AEI-1999-16

PUPT-1843

\title{
Radiation from Accelerated Branes
}

\author{
Mohab Abou-Zeid ${ }^{1}$ and Miguel S. Costa2 2 \\ ${ }^{1}$ Max Planck Institut für Gravitationsphysik, Albert Einstein Institut, \\ Am Mühlenberg 1, D-14476 Golm, Germany \\ ${ }^{2}$ Joseph Henry Laboratories, Princeton University, \\ Princeton, New Jersey 08544, USA
}

\begin{abstract}
The radiation emitted by accelerated fundamental strings and D-branes is studied within the linear approximation to the supergravity limit of string theory. We show that scalar, gauge field and gravitational radiation is generically emitted by such branes. In the case where an external scalar field accelerates the branes, we derive a Larmor-type formula for the emitted scalar radiation and study the angular distribution of the outgoing energy flux. The classical radii of the branes are calculated by means of the corresponding Thompson scattering cross sections. Within the linear approximation, the interaction of the external scalar field with the velocity fields of the branes gives a contribution to the observed gauge field and gravitational radiation.
\end{abstract}

\footnotetext{
${ }^{a}$ abouzeid@aei-potsdam.mpg.de

${ }^{b}$ miguel@feynman.princeton.edu
} 


\section{Introduction}

In addition to fundamental strings, nonperturbative superstring theory contains various types of extended BPS objects which play a key role in the web of dualities that have been uncovered in recent years, notably NS solitons [1, 2, 3] and Dbranes [4, 5], which carry R-R charges. The theory also contains non-BPS solitonic states, some of which are stable [6].

Among the BPS states, the NS 5-branes appear to be rather complicated objects whose dynamics is poorly understood, mainly because no tractable CFT description is known for them. However, it has been argued that the dynamics of NS 5-branes is given by a new theory of self-dual closed strings in six dimensions. This formulation has proved remarkably fruitful in studying the BPS spectrum of compactified string and $\mathrm{M}$ theories [7], even though the nature of the posited six-dimensional string theory without gravity remains somewhat mysterious.

By contrast, D-branes admit a very simple CFT description, at least at weak coupling, as hypersurfaces where open strings can end [4]. This has made it possible to use world-sheet techniques to study various aspects of the dynamics of nonrelativistic as well as relativistic D-branes, and to perform explicit computations of scattering amplitudes involving D-branes. This includes processes in which fundamental closed strings scatter off a D-brane [8, 9, 10, 11, 12], as well as processes in which a D-brane scatter off an identical D-brane [13]. Furthermore, D-branes have a number of properties which suggest that they are small at weak string coupling $g_{s}$ [8, 14]: in string units, their R-R charge is proportional to $g_{s}$ which is small and their tension is proportional to $1 / g_{s}$ which is small compared to the tension of the NS solitons (proportional to $1 / g_{s}^{2}$ ). This suggests that, in scattering processes in which a lower-dimensional D-brane scatter off a higher-dimensional D-brane, the former can be viewed as a probe of sub-stringy distances in the background geometry of the latter, and much can be learned about the physics at distances shorter than the tendimensional string and even the Planck lengths from this viewpoint 15, 16. In this framework, there are two descriptions of the interactions between D-branes: the supergravity interaction mediated by massless closed strings, and the probe quantum field theory which gives the effective dynamics of the various open string sectors. The range of validity of the two descriptions are different: supergravity is reliable at distances large compared to the string scale, while sub-stringy effects are governed by the light open strings.

However, most studies of string and D-brane scattering generally neglect a num-

ber of dynamical effects such as D-brane recoil and acceleration effects [17, 18, 19]. The latter are also important in the studies of the structure of black holes using 
strings and D-branes as probes [16]. For example, if the probe is excited, or the black hole is non-extremal, then supersymmetry is broken and the long range forces on an originally static probe do not exactly cancel. The resulting probe acceleration is $a \sim G M$ where $G$ is the Newton constant and $M$ the mass of the black hole. For a D-brane background, this gives an acceleration $a \sim g_{s}$, which is small at weak string coupling. For an F-string, this gives $a \sim g_{s}^{2}$, which is even smaller. For the NS 5-brane, the acceleration is of order one.

From the above considerations, it is clear that a better understanding of brane acceleration effects, which so far seem to have attracted little attention, is required. The aim of this paper is to start exploring these effects and to compute the radiation produced by moving branes. This may turn out to be of import to the understanding of string and $\mathrm{M}$ theory dynamics. For example, it was argued in [20] that rigid fundamental strings can only be accelerated up to a certain value of the acceleration $a_{c}$ which is of the same order as, and possibly related to, the Hagedorn temperature $T_{H}$ of string theory. Beyond this value, the accelerated string appears to be unstable. It would be interesting to investigate whether this observation extends to dynamical strings and to other objects in string theory. In particular, this might suggest a maximal acceleration for the open strings tethered on a D-branel or the self-dual closed strings making up the world-volume of a NS 5-brane. Recall that the BornInfeld-type effective action for D-branes already implies the existence of a limiting world-volume electric field $E_{c r i t}=\left(2 \pi \alpha^{\prime} c\right)^{-1}$, which is in dual correspondence with the limiting space-time velocity $c$ [13].

In the absence of a second quantized theory of strings and branes, it is not clear how acceleration effects should be described in (quantum) string theory. At any rate, several aspects of the dynamics of accelerated strings and branes can be studied in the classical supergravity description. In this paper we will focus on radiation effects from moving branes in the linear approximation to the classical limit of string theory.

The fundamental string solution couples to the metric, the NS-NS antisymmetric 2 -form potential and the dilaton and so one expects an accelerated F-string to produce dilatonic, 'electric' NS-NS antisymmetric tensor field and gravitational waves. In addition to the metric and dilaton fields, D-branes couple to the R-R gauge fields of the type II superstrings, so accelerated D-branes can be expected to emit dilatonic, gravitational and R-R radiation. Likewise, we expect accelerated NS 5-branes to emit dilatonic, gravitational and 'magnetic' antisymmetric tensor field waves. The $(p, q)$ strings of the type IIB theory [21, 22] are expected to emit both 'electric' NS-NS antisymmetric tensor field radiation and R-R 2-form gauge field

\footnotetext{
${ }^{c}$ We would like to thank Michael Green for suggesting this possibility.
} 
radiation in addition to the dilatonic and gravitational radiation. We will be able to confirm some of these expectations by explicit calculation of the supergravity fields produced by accelerated strings and D-branes at large distances in the weak field approximation.

We will restrict ourselves to situations in which the accelerated string or brane can be thought of as a localised source propagating in a background which is a small deformation of flat space-time at weak string coupling. This is possible for F-strings

and D-branes, but not for NS 5-branes. In the latter case the space-time metric involves a harmonic function $H=1+\mu / r^{2}$ in the four-dimensional transverse space with $\mu$ a constant of order one in string units, and hence space-time is not flat at small $g_{s}$.

The organization of the paper is as follows. In section 2, we review the system of coupled supergravity-brane field equations which we wish to solve in the linear approximation. We shall consider a weak external scalar field whose effect is to accelerate the branes. The solutions for the scalar fields, the $p$-form gauge field and the metric fluctuation are studied in sections 3, 4 and 5, respectively. In the case of the scalar fields, we derive the energy flux of the emitted radiation and study its angular distribution. We calculate the corresponding Thompson scattering crosssections and obtain the classical radii of the F-string and $\mathrm{D} p$-branes, in agreement with expectations from tree level string diagrams. The differential equations obeyed by the gauge field and metric fluctuation are discussed. These can in principle be solved for the radiation fields, although we have not been able to do so explicitly. This is because the external scalar field responsible for accelerating the branes interacts with the velocity fields (i. e. those not depending on the brane acceleration), which gives a contribution to the observed gauge and gravitational radiation fields that is not negligible within our approximations. We make some comments about possible extensions of the present work in the final section.

\section{Coupled Supergravity-Brane Field Equations}

The effective action for string theory in the presence of a $p$-brane in the long wavelength limit has the general form

$$
S=S_{\text {bulk }}+S_{\text {brane }}
$$

where $S_{b u l k}$ is the appropriate supergravity action and $S_{\text {brane }}$ is the effective action for the dynamics of the world-volume fields on the brane. In the following sections, we will consider the field equations derived from the bosonic part of the effective action (2.1) which describe the long-range bosonic fields outside a fundamental string 
or a $\mathrm{D} p$-brane source. At weak string coupling $g_{s}$, the corresponding background geometries are nearly flat and it is consistent to work in an approximation in which the branes are described by a sharply localised source propagating in a flat background. Our strategy will be to study the linearised theory around flat Minkowski space-time and to solve for the propagating small fluctuations of the NS-NS and (for the D-branes) the R-R fields.

Here we start by writing the bosonic bulk action for a 4-dimensional space-time obtained by compactification of 10-dimensional space-time on a rectangular 6-torus $T^{6}=T^{p} \times T^{(6-p)}$ : the spatial directions along the $p$-brane are wrapped around $T^{p}$, while $(6-p)$ of the remaining transverse directions are compactified on $T^{(6-p)}$. Then we derive the form of the bosonic particle actions arising from the compactified $p$-brane actions, and obtain the field equations of the coupled system. For branes accelerated by an external scalar field, we derive the linearised field equations.

\subsection{Compactified Effective Action}

For the fundamental string, the non-zero bulk fields correspond to the bosonic sector of the 3 -form version of $D=10, N=1$ supergravity. The string frame action is

$$
S_{\text {bulk }}=\frac{1}{2 \kappa_{10}^{2}} \int d^{10} x \sqrt{-G} e^{-2 \phi_{10}}\left(R+4\left(\partial_{a} \phi_{10}\right)^{2}-\frac{1}{2 \cdot 3 !} \mathcal{H}^{2}\right),
$$

where $a, b, \ldots$ are ten-dimensional space-time indices and $\mathcal{H}=d \mathcal{B}$ with $\mathcal{B}$ the NS-NS 2 -form gauge field. The gravitational coupling $\kappa_{10}$ is given by $2 \kappa_{10}^{2}=(2 \pi)^{7} g_{s}^{2} \alpha^{\prime 4}$. The ten-dimensional dilaton field $\phi_{10}$ has its zero mode substracted.

For the $\mathrm{D} p$-branes the appropriate bosonic bulk action corresponding to a truncation of the $N=2$ supergravities is

$$
S_{\text {bulk }}=\frac{1}{2 \kappa_{10}^{2}} \int d^{10} x \sqrt{-G}\left[e^{-2 \phi_{10}}\left(R+4\left(\partial_{a} \phi_{10}\right)^{2}\right)-\frac{1}{2 \cdot(p+2) !} \mathcal{F}_{p+2}^{2}\right],
$$

where $\mathcal{F}_{p+2}=d \mathcal{A}_{p+1}$ and $\mathcal{A}_{p+1}$ is the $(p+1)$-form $\mathrm{R}$ - $\mathrm{R}$ gauge field. For $p=3$, the field equations of the action (2.3) must be supplemented with the self-duality condition $\mathcal{F}_{5}=\star \mathcal{F}_{5}$, and for $p>3$ we consider the field strength $\mathcal{F}_{p+2}$ dual to $\mathcal{F}_{8-p}$.

We shall be interested in the overall motion of a wrapped brane in the uncompactified 4-dimensional space-time. In other words, we assume that the brane does not oscillate in the internal directions, and we neglect all of its worldvolume dynamics (except for a special case described below). Thus, we consider the situation in which the brane looks like a particle in the 4-dimensional space-time. It would be interesting to extend our analysis to the case of dynamical extended branes in ten 
uncompactified dimensions, including the world-volume excitations. We take $Y^{a}\left(\sigma^{\alpha}\right)$ as the ten-dimensional target space coordinates and work in the static gauge

$$
\begin{aligned}
& Y^{\mu}=Y^{\mu}(\tau), \quad \mu=0,1,2,3, \\
& Y^{\hat{m}}=\text { const. }, \quad \hat{m}=4, \ldots, 9-p \text {, } \\
& Y^{m}=\sigma^{m-(9-p)}, \quad m=10-p, \ldots, 9 \text {, }
\end{aligned}
$$

where $\sigma^{\alpha}$ are the worldvolume coordinates with $\alpha, \beta, \ldots=0, \ldots, p$. We neglect all the Kałuża-Klein modes in the compact and transverse directions $x^{\hat{m}}$. In the case of a compactified Dp-brane, our discussion includes the possibility of a world-line $U(1)$ gauge field $a_{\alpha}=a_{\alpha}(\tau)$ with an almost constant field strength $f=d a$. Such a gauge field corresponds to a slowly time-varying electric field $E_{\alpha}=\partial_{\tau} a_{\alpha}$ on the particle world-line. The T-dual system is a D-brane moving with velocity $v_{\alpha}=E_{\alpha}$. Thus, we can set $a_{\alpha}=0$ without restricting the generality: a T-duality transformation on the solution describing a particle in nearly uniform motion with velocity $v_{\alpha}$ yields the case of a slowly varying electric field $E_{\alpha}=v_{\alpha}$. The upper bound $E_{\text {crit }}$ on the magnitude of $E_{\alpha}$ is related to the limiting velocity $c$ of the particle. The framedependent magnitude of the velocity is otherwise unrestricted.

With the above assumptions it is consistent to compactify the ten-dimensional space-time to 4 dimensions using the following truncation

$$
\begin{gathered}
d s_{10}^{2}=e^{2 \phi} d s_{4}^{2}+e^{2 \lambda} d x^{\hat{m}} d x_{\hat{m}}+e^{2 \nu} d x^{m} d x_{m}, \\
\phi=\phi_{10}-\frac{1}{4} \ln \hat{G}=\phi_{10}-\frac{1}{2}[(6-p) \lambda+p \nu], \\
\mathcal{H}=F_{\mu \nu} d x^{\mu} \wedge d x^{\nu} \wedge d x^{9} \quad \text { or } \quad \mathcal{F}=F_{\mu \nu} \epsilon_{m_{1} \ldots m_{p}} d x^{\mu} \wedge d x^{\nu} \wedge d x^{m_{1}} \wedge \ldots \wedge d x^{m_{p}},
\end{gathered}
$$

where $\hat{G}=\operatorname{det}\left(G_{\hat{m} \hat{n}}\right) \cdot \operatorname{det}\left(G_{m n}\right)=e^{2(6-p) \lambda+2 p \nu}$. The field $\phi$ is the dilaton field of the compactified theory and $d s_{4}$ is the line element in the Einstein frame. The bulk actions (2.2) and (2.3) reduce to

$$
S_{\text {bulk }}=\frac{1}{2 \kappa^{2}} \int d^{4} x \sqrt{-g}\left[R-2\left(\partial_{\mu} \phi\right)^{2}-(6-p)\left(\partial_{\mu} \lambda\right)^{2}-p\left(\partial_{\mu} \nu\right)^{2}-\frac{1}{4} f(\phi, \lambda, \nu) F^{2}\right],
$$

where $\kappa^{2}=\kappa_{10}^{2} / V_{6}$ defines the 4 -dimensional gravitational coupling with $V_{6}$ the volume of the internal space and $f(\phi, \lambda, \nu)=e^{-a(p) \phi-b(p) \lambda-c(p) \nu}$ with

$$
\begin{array}{llll}
\text { F-string : } & a(1)=2, & b(1)=0, & c(1)=2, \\
\text { D p-brane: } & a(p)=0, & b(p)=-(6-p), & c(p)=p ;
\end{array}
$$

see e. g. 23] for a discussion of the reduction. 
In the case of the fundamental string $(p=1)$, the action (2.6) admits the solution 24]

$$
\begin{gathered}
d s_{4}^{2}=-H^{-\frac{1}{2}} d t^{2}+H^{\frac{1}{2}} d s^{2}\left(\mathbb{E}^{3}\right), \\
e^{2 \phi}=H^{-\frac{1}{2}}, \quad e^{2 \lambda}=1, \quad e^{2 \nu}=H^{-1}, \quad F=d H^{-1} \wedge d t
\end{gathered}
$$

where in the single centered case $H=1+\mu_{F} / r$. The constant $\mu_{F}$ is given by

$$
\mu_{F}=\frac{2 \kappa^{2}}{\Omega_{2}} M=\frac{2 \kappa^{2}}{\Omega_{2}} m T_{F} V_{1}=m \frac{(2 \pi)^{6} g_{s}^{2} \alpha^{\prime 3}}{\Omega_{2} V_{5}},
$$

where $\Omega_{2}=4 \pi$ denotes the area of the unit 2-sphere, $m$ is the string winding number, $V_{1}=2 \pi R_{1}$ is the length of the wrapped string and $V_{5}$ is the volume of the transverse compactified space. The fundamental string tension is $T_{F}=\left(2 \pi \alpha^{\prime}\right)^{-1}$. For the $\mathrm{D} p$-branes the solution takes the form [25]

$$
\begin{gathered}
d s_{4}^{2}=-H^{-\frac{1}{2}} d t^{2}+H^{\frac{1}{2}} d s^{2}\left(\mathbb{E}^{3}\right), \\
e^{2 \phi}=1, \quad e^{2 \lambda}=H^{\frac{1}{2}}, \quad e^{2 \nu}=H^{-\frac{1}{2}}, \quad F=d H^{-1} \wedge d t,
\end{gathered}
$$

where $H=1+\mu_{p} / r$. The constant $\mu_{p}$ is given by

$$
\mu_{p}=\frac{2 \kappa^{2}}{\Omega_{2}} M=\frac{2 \kappa^{2}}{\Omega_{2}} N T_{p} V_{p}=N \frac{(2 \pi)^{7-p} g_{s} \alpha^{\prime \frac{7-p}{2}}}{\Omega_{2} V_{6-p}},
$$

where $N$ is the number of D-branes or the D-brane winding number, and the $\mathrm{D} p$ brane tension is $T_{p}=\left((2 \pi)^{p} \alpha^{\prime \frac{p+1}{2}} g_{s}\right)^{-1}$.

Next we consider the action for the brane source. In the case of the fundamental string, we start with the bosonic action

$$
S_{F}=-\frac{T_{F}}{2} \int d^{2} \sigma\left(\sqrt{-\gamma} \gamma^{\alpha \beta} \partial_{\alpha} Y^{a} \partial_{\beta} Y^{b} G_{a b}+\epsilon^{\alpha \beta} \partial_{\alpha} Y^{a} \partial_{\beta} Y^{b} \mathcal{B}_{a b}\right)
$$

With the Ansätze (2.4) and (2.5) this action yields the following particle action:

$$
S=-M \int d \tau\left[\frac{1}{2}\left(-e^{2 \phi} U^{\mu} U^{\nu} g_{\mu \nu}+e^{2 \nu}\right)+U^{\mu} C_{\mu}\right]
$$

where $d C=F$ and we have defined the transverse 4-velocity vector

$$
U^{\mu}(\tau) \equiv \frac{d Y^{\mu}}{d \tau}
$$

In the case of a $\mathrm{D} p$-brane, we start with the effective action

$$
\begin{gathered}
S_{D p}=-T_{p} \int d^{p+1} \sigma\left(e^{-\phi_{10}} \sqrt{-\operatorname{det}\left(\hat{G}_{\alpha \beta}+2 \pi \alpha^{\prime} f_{\alpha \beta}-\hat{\mathcal{B}}_{\alpha \beta}\right)}\right. \\
\left.+\frac{1}{(p+1) !} \epsilon^{\alpha_{1} \ldots \alpha_{p+1}} \hat{\mathcal{A}}_{\alpha_{1} \ldots \alpha_{p+1}}\right)
\end{gathered}
$$


where $\hat{G}_{\alpha \beta}, \hat{\mathcal{B}}_{\alpha \beta}$ and $\hat{\mathcal{A}}_{\alpha_{1} \ldots \alpha_{p+1}}$ are the pull-backs to the worldvolume of the tendimensional bulk fields $G_{a b}, \mathcal{B}_{a b}$ and $\mathcal{A}_{a_{1} \ldots a_{p+1}}$, respectively. Note that the expansion of the Dirac-Born-Infeld part of the action around flat space is of second order in $\mathcal{B}_{\alpha \beta}$. Thus, in the linearised approximation considered in this paper we can set this field to zero. Also, as explained above, we can set $f_{\alpha \beta}=0$. The Dirac-Born-Infeld part of the action becomes

$$
\begin{aligned}
S & =-T_{p} \int d^{p+1} \sigma e^{-\phi_{10}} \sqrt{-\operatorname{det}\left(\hat{G}_{\alpha \beta}\right)} \\
& =-\frac{T_{p}}{2} \int d^{p+1} \sigma e^{-\phi_{10}} \sqrt{-\gamma}\left(\gamma^{\alpha \beta} \partial_{\alpha} Y^{a} \partial_{\beta} Y^{b} G_{a b}-(p-1)\right) .
\end{aligned}
$$

Note that, in the effective action (2.15), we have not included the generalized WessZumino coupling of the form $\mathcal{A} \exp \mathcal{F}$ [26, 27. This is consistent with our assumptions. For example, in the case of a time-varying electric field we should consider terms of the form $f \wedge \mathcal{A}_{p-1}$, and the $\mathrm{D} p$-brane would emit $\mathcal{A}_{p-1}$ gauge field radiation. Also, we have neglected certain anomalous couplings [28] and curvature terms [29]

which will play no role in the analysis of the present paper. With the Ansätze (2.4) and (2.5) we obtain the following particle action for the wrapped D $p$-brane

$$
S=-M \int d \tau\left[\frac{1}{2} e^{-\frac{6-p}{2} \lambda+\phi-\frac{p}{2} \nu}\left(-U^{\mu} U^{\nu} g_{\mu \nu}+p e^{-2 \phi+2 \nu}-(p-1) e^{-2 \phi}\right)+U^{\mu} C_{\mu}\right] .
$$

\section{$2.2 \quad$ Field Equations}

Here we give the bosonic field equations for the coupled supergravity-brane system. Consider first the field equations for the scalars. In the case of the F-string, the field equation for the dilaton field is

$$
\square \phi+\frac{1}{8} f(\phi, \nu) F^{2}=\frac{1}{2} \kappa^{2} M \int d \tau e^{2 \phi} \frac{\delta^{(4)}(x-Y(\tau))}{\sqrt{-g}},
$$

while in the case of the $\mathrm{D} p$-brane it takes the form

$$
\square \phi=\frac{1}{4} \kappa^{2} M \int d \tau e^{-\frac{6-p}{2} \lambda+\phi-\frac{p}{2} \nu}\left(1-p e^{-2 \phi+2 \nu}+(p-1) e^{-2 \phi}\right) \frac{\delta^{(4)}(x-Y(\tau))}{\sqrt{-g}} .
$$

The field equation for the scalar $\nu$ in the case of the F-string is

$$
\square \nu+\frac{1}{4} f(\phi, \nu) F^{2}=\kappa^{2} M \int d \tau e^{2 \nu} \frac{\delta^{(4)}(x-Y(\tau))}{\sqrt{-g}},
$$


while in the case of the $\mathrm{D} p$-brane it is

$$
\begin{gathered}
\square \nu+\frac{1}{8} f(\lambda, \nu) F^{2}= \\
\frac{1}{4} \kappa^{2} M \int d \tau e^{-\frac{6-p}{2} \lambda+\phi-\frac{p}{2} \nu}\left(-1+(4-p) e^{-2 \phi+2 \nu}+(p-1) e^{-2 \phi}\right) \frac{\delta^{(4)}(x-Y(\tau))}{\sqrt{-g}} .
\end{gathered}
$$

In the case of the F-string, the field equation for the scalar field $\lambda$ simplifies to

$$
\square \lambda=0
$$

while in the case of the $\mathrm{D} p$-brane it reads

$$
\begin{gathered}
\square \lambda-\frac{1}{8} f(\lambda, \nu) F^{2}= \\
-\frac{1}{4} \kappa^{2} M \int d \tau e^{-\frac{6-p}{2} \lambda+\phi-\frac{p}{2} \nu}\left(1+p e^{-2 \phi+2 \nu}-(p-1) e^{-2 \phi}\right) \frac{\delta^{(4)}(x-Y(\tau))}{\sqrt{-g}} .
\end{gathered}
$$

The gauge field equation for both the F-string and the Dp-brane is

$$
\partial_{\mu}\left(\sqrt{-g} f(\phi, \lambda, \nu) F^{\mu \nu}\right)=2 \kappa^{2} M \int d \tau U^{\nu}(\tau) \delta^{(4)}(x-Y(\tau)) .
$$

Finally, the Einstein equations read

$$
R^{\mu \nu}-\frac{1}{2} g^{\mu \nu} R=\kappa^{2}\left(T_{\phi}^{\mu \nu}+T_{\nu}^{\mu \nu}+T_{C}^{\mu \nu}+T_{\lambda}^{\mu \nu}+T_{\text {source }}^{\mu \nu}\right)
$$

where

$$
\begin{aligned}
\kappa^{2} T_{\phi}^{\mu \nu} & =2\left(\partial^{\mu} \phi \partial^{\nu} \phi-\frac{1}{2} g^{\mu \nu}(\partial \phi)^{2}\right), \\
\kappa^{2} T_{\nu}^{\mu \nu} & =p\left(\partial^{\mu} \nu \partial^{\nu} \nu-\frac{1}{2} g^{\mu \nu}(\partial \nu)^{2}\right), \\
\kappa^{2} T_{\lambda}^{\mu \nu} & =(6-p)\left(\partial^{\mu} \lambda \partial^{\nu} \lambda-\frac{1}{2} g^{\mu \nu}(\partial \lambda)^{2}\right), \\
\kappa^{2} T_{C}^{\mu \nu} & =\frac{1}{2} f(\phi, \lambda, \nu)\left(F_{\eta}^{\mu} F^{\nu \eta}-\frac{1}{4} g^{\mu \nu} F^{2}\right),
\end{aligned}
$$

are the contributions of the scalar fields and of the gauge field to the stress-energy tensor derived from (2.6). The stress-energy tensors of the sources are given by

$$
\begin{array}{ll}
\text { F-string : } & T_{\text {source }}^{\mu \nu}=M \int d \tau U^{\mu}(\tau) U^{\nu}(\tau) e^{2 \phi} \frac{\delta^{(4)}(x-Y(\tau))}{\sqrt{-g}} \\
\text { D } p \text {-brane }: & T_{\text {source }}^{\mu \nu}=M \int d \tau U^{\mu}(\tau) U^{\nu}(\tau) e^{-\frac{6-p}{2} \lambda+\phi-\frac{p}{2} \nu} \frac{\delta^{(4)}(x-Y(\tau))}{\sqrt{-g}} .
\end{array}
$$

\subsection{Accelerated Branes}

For small string coupling (and $r$ large enough), the supergravity solutions (2.8) and (2.10) can be written as an expansion in $\left(\kappa^{2} M\right)$ around flat space. Then, we 
may regard the branes as delta-function sources localised at $r=0$. In order to accelerate the branes we further consider some weak external field. For simplicity we consider an external scalar field and to be definite we restrict ourselves to the scalar field $\nu$. If $\nu_{0}(x)$ is the external accelerating field we write

$$
\nu(x) \rightarrow \nu_{0}(x)+\nu(x)
$$

where $\nu_{0}(x)$ admits some Fourier decomposition $\nu_{0}(x)=\int d^{4} k \nu_{0}(k) e^{-i k \cdot x}$ and the field $\nu(x)$ represents the scalar field generated by the brane source.

To leading order in the external field $\nu_{0}(x)$ the equation of motion for the F-string is

$$
\dot{U}^{\mu}+\partial^{\mu} \nu_{0}=0
$$

where $\dot{U} \equiv d U / d \tau$ is the 4 -acceleration. For the $\mathrm{D} p$-brane, the corresponding equation is

$$
\dot{U}^{\mu}+\frac{p}{2}\left(-U^{\mu} U \cdot \partial \nu_{0}+\partial^{\mu} \nu_{0}\right)=0
$$

In both cases, taking the scalar product of the 4-velocity with the corresponding equation of motion, we conclude that $U \cdot \partial \nu_{0}=0$. Thus, (2.29) simplifies to

$$
\dot{U}^{\mu}+\frac{p}{2} \partial^{\mu} \nu_{0}=0
$$

Also, note that the acceleration is of first order in $\nu_{0}$, i.e. $\dot{U} \sim \nu_{0}$. In the nonrelativistic limit the equations of motion for the F-string and $\mathrm{D} p$-brane become

$$
\dot{\mathbf{v}}+\partial \nu_{0}=0 \quad \text { and } \quad \dot{\mathbf{v}}+\frac{p}{2} \partial \nu_{0}=0
$$

respectively.

\subsection{Linearised Field Equations}

Since the fields generated by the brane source can be written as an expansion in $\left(\kappa^{2} M\right)$, we shall work to first order in this expansion parameter and keep only the terms linear in such fields. In other words, the source terms in the field equations of section 2.2 are taken to be small. Also, we assume that the external field $\nu_{0}(x)$ is weak and keep only the terms linear in this accelerating field. Since the magnitude $a$ of the acceleration is limited on dimensional grounds to $a \ll 1 / \sqrt{\alpha^{\prime}}$ in order for the higher in $\alpha^{\prime}$ derivative corrections to supergravity to be negligible, the external field $\nu_{0}(x)$ must satisfy $\left(\partial \nu_{0}\right)^{2} \ll 1 / \alpha^{\prime}$. In the case of D-branes, the upper bound $1 / \sqrt{\alpha^{\prime}}$ on the magnitude of the acceleration also ensures that corrections due to derivatives of the world-volume fields (in particular derivatives of the scalars $Y$ ) to the effective brane action can be consistently neglected. 
In the case of the F-string, the linearised field equations for the scalar fields take the form

$$
\begin{gathered}
\partial^{2} \phi=\frac{1}{2} \kappa^{2} M \int d \tau \delta^{(4)}(x-Y(\tau)), \\
\partial^{2} \nu=\kappa^{2} M \int d \tau\left(1+2 \nu_{0}(Y(\tau))\right) \delta^{(4)}(x-Y(\tau)), \\
\partial^{2} \lambda=0 .
\end{gathered}
$$

In the case of the $\mathrm{D} p$-brane we find

$$
\begin{gathered}
\partial^{2} \phi=\frac{1}{2} \kappa^{2} M \int d \tau\left(-p \nu_{0}(Y(\tau))\right) \delta^{(4)}(x-Y(\tau)), \\
\partial^{2} \nu=\frac{1}{2} \kappa^{2} M \int d \tau\left(1+\left(4-\frac{3}{2} p\right) \nu_{0}(Y(\tau))\right) \delta^{(4)}(x-Y(\tau)), \\
\partial^{2} \lambda=-\frac{1}{2} \kappa^{2} M \int d \tau\left(1+\frac{p}{2} \nu_{0}(Y(\tau))\right) \delta^{(4)}(x-Y(\tau)) .
\end{gathered}
$$

The linearised gauge field equation for both the F-string and the Dp-brane is given by

$$
\partial_{\mu}\left(\left(1-c(p) \nu_{0}(x)\right) F^{\mu \nu}\right)=2 \kappa^{2} M \int d \tau U^{\nu}(\tau) \delta^{(4)}(x-Y(\tau)) .
$$

Finally, we give the field equations for the metric fluctuations. In the case of the F-string, we find

$$
\begin{aligned}
\partial^{2} \bar{h}^{\mu \nu}= & -2 \kappa^{2} M \int d \tau U^{\mu}(\tau) U^{\nu}(\tau) \delta^{(4)}(x-Y(\tau)) \\
& -2 p\left(2 \partial^{(\mu} \nu_{0} \partial^{\nu)} \nu-\eta^{\mu \nu} \partial \nu_{0} \cdot \partial \nu\right)
\end{aligned}
$$

where the metric was split as $g_{\mu \nu}=\eta_{\mu \nu}+h_{\mu \nu}$ and the definition $\bar{h}_{\mu \nu} \equiv h_{\mu \nu}-\frac{1}{2} \eta_{\mu \nu} h$ with $h \equiv h_{\mu}^{\mu}$ was introduced. The tensor $\bar{h}_{\mu \nu}$ is taken to satisfy the gauge condition

$$
\partial_{\mu} \bar{h}^{\mu \nu}=0 .
$$

In the case of the $\mathrm{D} p$-brane, the corresponding equation is

$$
\begin{aligned}
\partial^{2} \bar{h}^{\mu \nu}= & -2 \kappa^{2} M \int d \tau U^{\mu}(\tau) U^{\nu}(\tau)\left(1-\frac{p}{2} \nu_{0}(Y(\tau))\right) \delta^{(4)}(x-Y(\tau)) \\
& -2 p\left(2 \partial^{(\mu} \nu_{0} \partial^{\nu)} \nu-\eta^{\mu \nu} \partial \nu_{0} \cdot \partial \nu\right)
\end{aligned}
$$

To understand physically the various terms in the above equations, consider equation (2.38). The first line on the right hand side has two parts: the factor of 
1 inside the bracket gives a source term for the brane, while the factor involving $\nu_{0}(Y(\tau))$ governs the scattering of the external field by the brane source, i.e. the absorption of closed strings with the quantum numbers of the field $\nu_{0}(x)$ and the emission of closed strings with those of the graviton. The second line on the right hand side of (2.38) governs the interaction of the external field $\nu_{0}(x)$ with the field $\nu$ generated by the brane source, which results in the emission of gravitons.

\section{Scalar Radiation from Accelerated Branes}

In this section we study the scalar field radiation emitted by accelerated branes. There are three different scalar fields, namely the dilaton $\phi$ and the compactification scalars $\lambda$ and $\nu$. For the sake of brevity, we will only consider the scalar field $\nu$; similar results hold for the other scalars. The results obtained below are generalizations of standard results in classical electrodynamics; for a more detailed discussion, see e.g. 30], in particular chapter 14. We start by finding a solution to the field equation for the scalar field $\nu$. Next we compute the energy flux of the emitted radiation and study its angular dependence. This result is used to calculate the Thompson scattering cross sections for the scalar $\nu$, from which the classical radii of the branes are deduced.

The retarded solution to the linearised field equations (2.33) and (2.34) for the scalar $\nu(x)$ can be written in the integral form

$$
\nu(x)=\int d^{D} x^{\prime} D_{r}\left(x-x^{\prime}\right) J\left(x^{\prime}\right),
$$

where $D_{r}\left(x-x^{\prime}\right)$ is the retarded Green function,

$$
D_{r}\left(x-x^{\prime}\right)=-\frac{1}{2 \pi} \theta\left(x^{0}-x^{\prime 0}\right) \delta\left[\left(x-x^{\prime}\right)^{2}\right]
$$

(here $\theta\left(x_{0}\right)$ is the usual step function satisfying $\theta\left(x_{0}\right)=0$ for $x_{0}<0$ and $\theta\left(x_{0}\right)=1$ for $\left.x_{0}>0\right)$, and

$$
J\left(x^{\prime}\right)=A \kappa^{2} M \int d \tau\left(1+d(p) \nu_{0}(Y(\tau))\right) \delta^{(4)}\left(x^{\prime}-Y(\tau)\right),
$$

with

$$
\begin{array}{lll}
\text { F-string : } & A=1, & d(1)=2, \\
\text { D } p \text {-brane : } & A=\frac{1}{2}, & d(p)=4-\frac{3}{2} p .
\end{array}
$$

The retarded Green function (3.2) is non-zero only on the forward light-cone of the source point, a requirement that leads to the solution

$$
\nu(x)=\frac{A \kappa^{2} M}{4 \pi}\left[\frac{1+d(p) \nu_{0}(Y(\tau))}{U \cdot(x-Y(\tau))}\right]_{\tau=\tau_{0}},
$$


where $\tau_{0}$ is defined by the light cone condition

$$
\left[x-Y\left(\tau_{0}\right)\right]^{2}=0
$$

and the retardation requirement $x^{0}>Y^{0}\left(\tau_{0}\right)$. The light cone condition is equivalent to

$$
x^{0}-Y^{0}\left(\tau_{0}\right)=\left|\mathbf{x}-\mathbf{Y}\left(\tau_{0}\right)\right| \equiv R
$$

\subsection{Radiated Power}

To calculate the power radiated by the brane in the form of waves of the scalar field $\nu$, we define the Poynting vector $\mathbf{S}$ by $S^{i}=T_{\nu}^{0 i}$, where $T_{\nu}^{\mu \nu}$ is the contribution of this scalar field to the stress-energy tensor given in (2.26). In the linear approximation,

$$
S_{\nu}^{i}=T_{\nu}^{0 i}=\frac{p}{\kappa^{2}}\left(\partial^{0} \nu\right)\left(\partial^{i} \nu\right)
$$

Notice that we assume the incident beam $\nu_{0}(x)$ to be well localised such that it does not mix with the emitted radiation far from the source (otherwise there would be terms proportional to $(\partial \nu)\left(\partial \nu_{0}\right)$ in the Poynting vector).

To calculate the Poynting vector we have to compute the derivatives with respect to the observation coordinates $x \equiv(t, \mathbf{x})$ of the retarded quantity (3.5). In doing so it is important to realize that functions of the proper time $\tau$ depend implicitly on $x$ through the relation (3.6) 31]. For a general function $f(x, \tau)$ we have

$$
\partial^{\mu} f=\left.\partial^{\mu} f\right|_{\tau}+\partial^{\mu} \tau \frac{d f}{d \tau}
$$

where

$$
\partial^{\mu} \tau=\frac{(x-Y(\tau))^{\mu}}{U \cdot(x-Y(\tau))}
$$

Thus, the derivative of the scalar field $\nu$ is

$$
\begin{aligned}
\partial^{\mu} \nu= & \frac{A \kappa^{2} M}{4 \pi} \frac{1}{[U \cdot(x-Y(\tau))]^{2}}\left\{[ 1 + d ( p ) \nu _ { 0 } ( Y ( \tau ) ) ] \left(-\frac{(x-Y(\tau))^{\mu}}{U \cdot(x-Y(\tau))}\right.\right. \\
& \left.\left.-U^{\mu}-\frac{(x-Y(\tau))^{\mu}}{U \cdot(x-Y(\tau))} \dot{U} \cdot(x-Y(\tau))\right)+(x-Y(\tau))^{\mu} d(p) \partial \nu_{0} \cdot U\right\}
\end{aligned}
$$

evaluated at the retarded time $\tau=\tau_{0}$. The last term in this equation vanishes because $\partial \nu_{0} \cdot U=0$. It is convenient to write $\partial^{\mu} \nu$ in non-covariant form using the formulae

$$
\begin{aligned}
(x-Y)^{\mu} & \equiv R(1, \mathbf{n}), \quad U^{\mu}=\gamma(1, \mathbf{v}), \quad \gamma^{2}=\frac{1}{1-\mathbf{v}^{2}} \\
\dot{U}^{\mu} & \equiv \frac{d U^{\mu}}{d \tau}=\gamma^{2}\left(\gamma^{2} \mathbf{v} \cdot \dot{\mathbf{v}}, \dot{\mathbf{v}}+\gamma^{2} \mathbf{v}(\mathbf{v} \cdot \dot{\mathbf{v}})\right)
\end{aligned}
$$


where $\mathbf{n}$ is the unit vector in the direction of observation $\mathbf{x}-\mathbf{Y}(\tau)$ and $\dot{\mathbf{v}}=d \mathbf{v} / d Y^{0}$. The result is

$$
\begin{array}{r}
\partial \nu=\frac{A \kappa^{2} M}{4 \pi}\left(1+d(p) \nu_{0}(Y(\tau))\right)\left[\frac{(1, \mathbf{n})}{R^{2} \gamma^{3}(1-\mathbf{n} \cdot \mathbf{v})^{3}}-\frac{(1, \mathbf{v})}{R^{2} \gamma(1-\mathbf{n} \cdot \mathbf{v})^{2}}\right. \\
\left.-\frac{(1, \mathbf{n})}{R \gamma(1-\mathbf{n} \cdot \mathbf{v})^{2}}\left(\gamma^{2}(\mathbf{v} \cdot \dot{\mathbf{v}})-\frac{\mathbf{n} \cdot \dot{\mathbf{v}}}{1-\mathbf{n} \cdot \mathbf{v}}\right)\right] .
\end{array}
$$

This divides naturally into 'velocity fields' and 'acceleration fields'. The first two terms in the square bracket are velocity fields which fall off as $1 / R^{2}$ and are static fields, as can be seen by performing a Lorentz transformation. The last term in the bracket contains the acceleration fields, which fall off as $1 / R$ and are typical radiation fields. Both the velocity and the acceleration fields are multiplied by an extra factor of $d(p) \nu_{0}(Y(\tau))$ which is induced by the external field. Keeping only the radiation fields, and noting that $\dot{U} \sim \nu_{0}$, we find to linear order

$$
\partial \nu=\frac{A \kappa^{2} M}{4 \pi}\left[-\frac{(1, \mathbf{n})}{R \gamma(1-\mathbf{n} \cdot \mathbf{v})^{2}}\left(\gamma^{2}(\mathbf{v} \cdot \dot{\mathbf{v}})-\frac{\mathbf{n} \cdot \dot{\mathbf{v}}}{1-\mathbf{n} \cdot \mathbf{v}}\right)\right] .
$$

Thus, $\partial^{i} \nu=\left(\partial^{0} \nu\right) n^{i}$ for the radiation fields and hence

$$
\mathbf{S}=\frac{p}{\kappa^{2}}\left(\partial^{0} \nu\right)^{2} \mathbf{n}
$$

For an accelerated brane in a frame where the velocity is small $(v \ll 1)$, we find

$$
\partial^{0} \nu=\frac{A \kappa^{2} M}{4 \pi} \frac{\mathbf{n} \cdot \dot{\mathbf{v}}}{R}
$$

which gives the Poynting vector

$$
\mathbf{S}=p\left(\frac{A \kappa M}{4 \pi R}\right)^{2}|\dot{\mathbf{v}}|^{2} \cos ^{2} \theta \mathbf{n}
$$

where $\theta$ is the angle between $\mathbf{v}$ and the observation direction $\mathbf{n}$. The power radiated per unit of solid angle is $\frac{d P(t)}{d \Omega}=R^{2}(\mathbf{S} \cdot \mathbf{n})$. Upon integrating over all solid angle we obtain the total (instantaneous) power radiated,

$$
P=\frac{p}{12 \pi}(A \kappa M)^{2}|\dot{\mathbf{v}}|^{2} .
$$

This is the Larmor formula for a (nonrelativistic) accelerated wrapped brane. The total power emitted by an accelerated wrapped brane in relativistic motion is given by the unique Lorentz invariant generalization of (3.18) obtained by substituting

$$
|\dot{\mathbf{v}}|^{2} \rightarrow \dot{U} \cdot \dot{U}=\gamma^{4}\left(\gamma^{2}(\mathbf{v} \cdot \dot{\mathbf{v}})^{2}+\dot{\mathbf{v}}^{2}\right)
$$



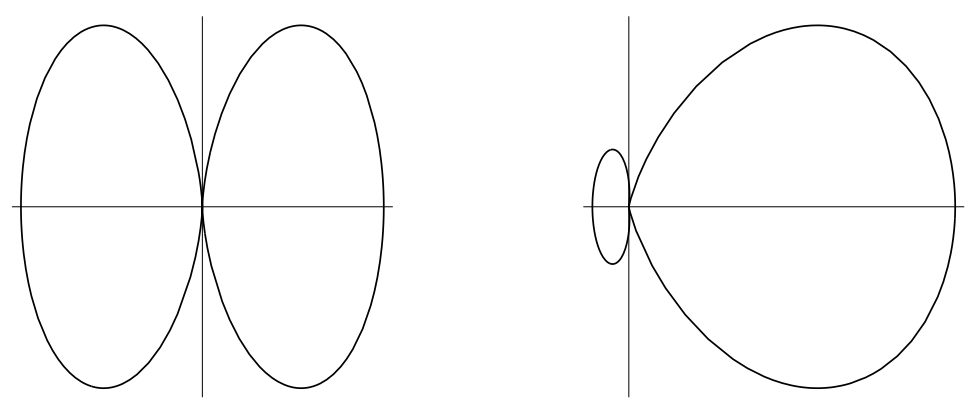

Figure 1: Angular distribution of the scalar field radiation for an accelerated brane with $\mathbf{v}$ and $\dot{\mathbf{v}}$ parallel in the non-relativistic (left) and the relativistic (right) regimes. Note that as $v \rightarrow 1$, the angular distribution is tipped forward and increases in magnitude.

\subsection{Angular Distribution of Radiation}

In terms of the proper time $t^{\prime}$ of the brane, the general formula for the power radiated in the form of waves of the scalar field $\nu$ per unit of solid angle is given by

$$
\frac{d P\left(t^{\prime}\right)}{d \Omega}=p\left(\frac{A \kappa M}{4 \pi}\right)^{2} \frac{\left[\gamma^{2}(1-\mathbf{n} \cdot \mathbf{v}) \mathbf{v} \cdot \dot{\mathbf{v}}-\mathbf{n} \cdot \dot{\mathbf{v}}\right]^{2}}{(1-\mathbf{n} \cdot \mathbf{v})^{5}}
$$

We consider two special cases of this result: in the first, $\mathbf{v}$ and $\dot{\mathbf{v}}$ are parallel, while in the second they are perpendicular. In the former situation, equation (3.20) becomes

$$
\frac{d P\left(t^{\prime}\right)}{d \Omega}=p\left(\frac{A \kappa M}{4 \pi}\right)^{2} \gamma^{2} \dot{v}^{2} \frac{(v-\cos \theta)^{2}}{(1-v \cos \theta)^{5}}
$$

For $v \ll 1$, this is the Larmor result. For $v \sim 1$, the angular distribution is tipped forward as indicated schematically in figure 1 .

If $\mathbf{v}$ and $\dot{\mathbf{v}}$ are perpendicular, then the angular distribution (3.20) becomes

$$
\frac{d P\left(t^{\prime}\right)}{d \Omega}=p\left(\frac{A \kappa M}{4 \pi}\right)^{2} \frac{\dot{v}^{2}}{\gamma^{2}} \frac{\sin ^{2} \theta \cos ^{2} \phi}{(1-v \cos \theta)^{5}},
$$

where we are using spherical coordinates such that $\mathbf{v} \cdot \mathbf{n}=v \cos \theta$ and $\dot{\mathbf{v}} \cdot \mathbf{n}=$ $\dot{v} \sin \theta \cos \phi$. The distribution in $\theta$ at fixed angle $\phi$ is shown schematically in figure 2 .

\subsection{Thompson Scattering}

The previous results can be used to calculate the cross section for the emission of waves of the scalar field $\nu$ (as well as radiation of $\phi$ and $\lambda$ waves). In the nonrelativistic limit the brane motion has the same frequency as the incident radiation 

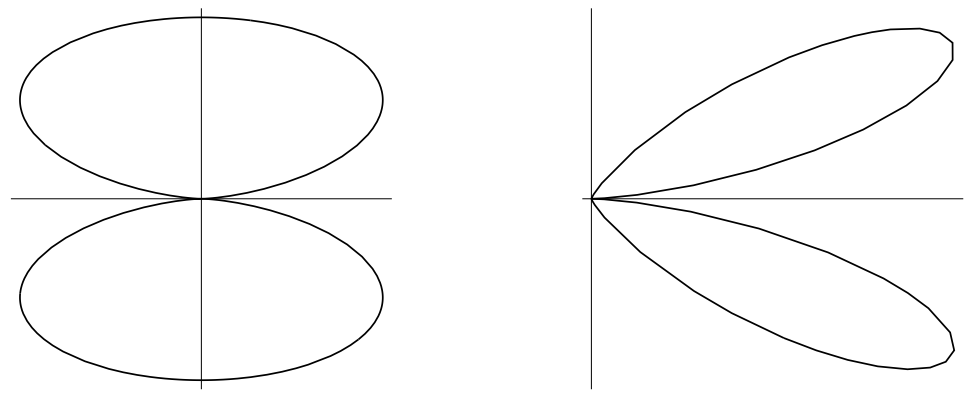

Figure 2: Angular distribution of the scalar field radiation for an accelerated brane with $\mathbf{v}$ and $\dot{\mathbf{v}}$ perpendicular at fixed angle $\phi$ (with $\cos \phi \neq 0$ ) in the non-relativistic (left) and the relativistic (right) regimes. As $v \rightarrow 1$, the distribution is tipped forward and the intensity reaches a maximum for a particular value of the angle, $\theta_{\text {max }}$. In the direction perpendicular to $\dot{\mathbf{v}}(\cos \phi=0)$, no radiation is observed.

and the process can be described as scattering of the incident radiation. For nonrelativistic motion, the instantaneous power per unit of solid angle radiated by the brane is

$$
\frac{d P}{d \Omega}=p\left(\frac{A \kappa M}{4 \pi}\right)^{2}|\dot{\mathbf{v}}|^{2} \cos ^{2} \theta .
$$

The incident wave $\nu_{0}(k) e^{-i k \cdot x}$ induces an acceleration

$$
\dot{\mathbf{v}}=-p A i \mathbf{k} \nu_{0}(k) e^{-i k \cdot x}
$$

In the long wave-length approximation, we can assume that the brane moves a negligible part of a wave-length during one oscillation and therefore the time average of $|\dot{\mathbf{v}}|^{2}$ is $\frac{1}{2} \operatorname{Re}\left(\dot{\mathbf{v}} \cdot \dot{\mathbf{v}}^{*}\right)$. Then, the average radiated power per unit of solid angle is

$$
\left\langle\frac{d P}{d \Omega}\right\rangle=p\left(\frac{A \kappa M}{4 \pi}\right)^{2} \frac{(p A)^{2}}{2}|\mathbf{k}|^{2}\left|\nu_{0}\right|^{2} \cos ^{2} \theta .
$$

Since the average incident flux for the plane wave is $\frac{p}{\kappa^{2}} \frac{1}{2}|\mathbf{k}|^{2}\left|\nu_{0}\right|^{2}$ we obtain the differential scattering cross section

$$
\frac{d \sigma}{d \Omega}=\left(\frac{p A^{2} \kappa^{2} M}{4 \pi}\right)^{2} \cos ^{2} \theta .
$$

This result is in agreement with expectations from consideration of tree level string diagrams. For the F-string, the scattering amplitude is proportional to $\left(\kappa^{2} M\right) \sim g_{s}^{2}$, corresponding to a sphere with four vertex operator insertions. In the case of the D $p$-brane, the amplitude is proportional to $\left(\kappa^{2} M\right) \sim g_{s}$, corresponding to a disk with two vertex operator insertions in the interior. 
Integrating the differential cross section (3.26) over all solid angle, we obtain the Thompson cross section for emission of waves of the scalar field $\nu$. The length scale $\kappa^{2} M$ defines the classical radii $R_{F}$ and $R_{p}$ of the $\mathrm{F}$-string and the $\mathrm{D} p$-branes:

$$
R_{F} \sim \kappa^{2} M_{F} \sim g_{s}^{2} \frac{\alpha^{\prime 3}}{V_{5}} \quad \text { and } \quad R_{p} \sim \kappa^{2} M_{p} \sim g_{s} \frac{\alpha^{\prime \frac{7-p}{2}}}{V_{6-p}}
$$

\section{Gauge Field Radiation from Accelerated Branes}

Consider the linearised field equation for the gauge field,

$$
\partial_{\mu}\left(\left(1-c(p) \nu_{0}(x)\right) F^{\mu \nu}\right)=2 \kappa^{2} M \int d \tau U^{\nu}(\tau) \delta^{(4)}(x-Y(\tau)) .
$$

In order to solve this coupled equation, we split the gauge field as $C^{\mu}=C_{(0)}^{\mu}+C_{(1)}^{\mu}$, where $C_{(0)}^{\mu}$ does not depend explicitly on $\nu_{0}$ and $C_{(1)}^{\mu}$ depends linearly on $\nu_{0}$. Working in the gauge

$$
\partial_{\mu} C^{\mu}=0
$$

and dropping quadratic terms in $\nu_{0}$, we find the pair of equations

$$
\begin{gathered}
\partial^{2} C_{(0)}^{\mu}=2 \kappa^{2} M \int d \tau U^{\nu}(\tau) \delta^{(4)}(x-Y(\tau)), \\
\partial^{2} C_{(1)}^{\mu}=c(p) \partial_{\mu}\left(\nu_{0}(x) F_{(0)}^{\mu \nu}\right) .
\end{gathered}
$$

As for the scalar fields, we use the retarded Green function (3.2) to find the solution for $C_{(0)}^{\mu}$. The result is

$$
C_{(0)}^{\mu}=\frac{\kappa^{2} M}{2 \pi}\left[\frac{U^{\nu}(\tau)}{U \cdot(x-Y(\tau))}\right]_{\tau=\tau_{0}}
$$

and the corresponding field strength is

$$
F_{(0)}^{\mu \nu}=\frac{\kappa^{2} M}{2 \pi} \frac{2(x-Y(\tau))^{[\mu}}{[U \cdot(x-Y(\tau))]^{2}}\left[-\frac{U^{\nu]}}{U \cdot(x-Y(\tau))}-\frac{U^{\nu]} \dot{U} \cdot(x-Y(\tau))}{U \cdot(x-Y(\tau))}+\dot{U}^{\nu]}\right]
$$

this expression must be evaluated at $\tau=\tau_{0}$. This is the same answer as in classical electrodynamics. Since $C_{(0)}^{\mu}$ obeys by itself the gauge condition $\partial_{\mu} C_{(0)}^{\mu}=0$, the field equation for $C_{(1)}^{\mu}$ becomes

$$
\partial^{2} C_{(1)}^{\mu}=c(p)\left(\left(\partial_{\mu} \nu_{0}\right) F_{(0)}^{\mu \nu}+\nu_{0}(x) \partial^{2} C_{(0)}^{\nu}\right)
$$

which can be written as

$$
\partial^{2} C_{(1)}^{\mu}=c(p)\left(\left(\partial_{\mu} \nu_{0}\right) F_{(0)}^{\mu \nu}+\nu_{0}(x) 2 \kappa^{2} M \int d \tau U^{\nu}(\tau) \delta^{(4)}(x-Y(\tau))\right) .
$$


Since $\dot{U} \sim \nu_{0}$, we may drop the acceleration terms in $F_{(0)}^{\mu \nu}$. Then, to first order in $\nu_{0}$ equation (4.7) simplifies to

$$
\begin{aligned}
\partial^{2} C_{(1)}^{\mu}=\frac{\kappa^{2} M}{2 \pi} c(p)[ & -\left(\partial_{\mu} \nu_{0}\right)\left(\frac{2(x-Y(\tau))^{[\mu} U^{\nu]}}{[U \cdot(x-Y(\tau))]^{3}}\right)_{\tau=\tau_{0}} \\
& \left.+4 \pi \int d \tau \nu_{0}(Y(\tau)) U^{\nu}(\tau) \delta^{(4)}(x-Y(\tau))\right] .
\end{aligned}
$$

Let us first consider the second term on the right hand side of this equation. The corresponding contribution to the field $C_{(1)}^{\mu}$ is

$$
C_{(1)}^{\mu} \sim \frac{\kappa^{2} M}{2 \pi} c(p)\left[\frac{\nu_{0}(Y(\tau)) U^{\nu}(\tau)}{U \cdot(x-Y(\tau))}\right]_{\tau=\tau_{0}}
$$

which gives a field strength

$$
F_{(1)}^{\mu \nu} \sim \frac{\kappa^{2} M}{2 \pi} c(p)\left[\frac{2\left(\partial \nu_{0} \cdot U\right)(x-Y(\tau))^{[\mu} U^{\nu]}}{[U \cdot(x-Y(\tau))]^{2}}\right]_{\tau=\tau_{0}}+\ldots
$$

where the ellipsis refers to velocity terms that are linear in $\nu_{0}$ but fall off as $1 / R^{2}$ and to acceleration terms that fall off as $1 / R$ but are quadratic in $\nu_{0}$. Since $\partial \nu_{0} \cdot U=0$ we conclude that the contribution to the observed radiation of the second term on the right hand side of (4.8) is negligible. Thus, within our approximations equation (4.8) reduces to

$$
\partial^{2} C_{(1)}^{\nu}=-\frac{\kappa^{2} M}{2 \pi} c(p)\left(\partial_{\mu} \nu_{0}\right)\left(\frac{2(x-Y(\tau))^{[\mu} U^{\nu]}}{[U \cdot(x-Y(\tau))]^{3}}\right)_{\tau=\tau_{0}} .
$$

Physically this equation describes the interaction of the external field $\nu_{0}(x)$ with the velocity fields in $C_{(0)}^{\mu}$. This generates the field $C_{(1)}^{\mu}$. The contribution of $C_{(1)}^{\mu}$ to the energy flux at infinity is not negligible. From equation (4.11), we conclude that $\partial^{2} C_{(1)}^{\nu} \sim \nu_{0} / R^{2}$ and therefore $\partial^{\mu} C_{(1)}^{\nu} \sim F_{(1)}^{\mu \nu} \sim \nu_{0} / R$. Thus the radiation gauge field has two different contributions. The first one, described by (4.4) and (4.5), corresponds to the usual result in electrodynamics. The second one can be obtained by solving the differential equations (4.2) and (4.11) and corresponds to the interaction of the external field with the velocity fields in $F_{(0)}^{\mu \nu}$. This additional contribution to the radiation fields stems from the non-linearity of the original system of coupled field equations: for example, unlike in electrodynamics, an external electromagnetic wave interacts with the bulk scalar, gauge and graviton fields of the source; this gives a contribution to the scalar, gauge field and gravitational radiation emitted.

Although we were not able to find a solution of the equations (4.2) and (4.11) in closed form, it is clear that these equations can be integrated. The solution should 
then be substituted in the stress-energy tensor for the gauge field given in eq. (2.26). The computations of the corresponding Poynting vector, of the differential and total power radiated and of the Thomson scattering cross section are then similar to those given in section 3, albeit more tedious.

\section{$5 \quad$ Gravitational Radiation from Accelerated Branes}

In this section we study the solution for the metric fluctuations. The linearised equation for $\bar{h}^{\mu \nu}$ in the case of the F-string was derived in section 2, with the result

$$
\begin{aligned}
\partial^{2} \bar{h}^{\mu \nu}= & -2 \kappa^{2} M \int d \tau U^{\mu}(\tau) U^{\nu}(\tau) \delta^{(4)}(x-Y(\tau)) \\
& -2 p\left(2 \partial^{(\mu} \nu_{0} \partial^{\nu)} \nu-\eta^{\mu \nu} \partial \nu_{0} \cdot \partial \nu\right)
\end{aligned}
$$

In the case of the $\mathrm{D} p$-branes, there is an extra term of $-\frac{p}{2} \nu_{0}(Y(\tau))$ multiplying the delta-function source; this we can ignore for reasons explained in section 3 when studying scalar field radiation. As in the case of the gauge field, we split the metric fluctuations as $\bar{h}^{\mu \nu}=\bar{h}_{(0)}^{\mu \nu}+\bar{h}_{(1)}^{\mu \nu}$ and obtain the equations

$$
\begin{aligned}
& \partial^{2} \bar{h}_{(0)}^{\mu \nu}=-2 \kappa^{2} M \int d \tau U^{\mu}(\tau) U^{\nu}(\tau) \delta^{(4)}(x-Y(\tau)) \\
& \partial^{2} \bar{h}_{(1)}^{\mu \nu}=2 p \frac{A \kappa^{2} M}{4 \pi} \frac{1}{[U \cdot(x-Y(\tau))]^{2}}\left(2 \frac{\left(\partial^{(\mu} \nu_{0}\right)(x-Y(\tau))^{\nu)}}{U \cdot(x-Y(\tau))}+2 \partial^{(\mu} \nu_{0}^{\nu)}\right. \\
&\left.-\eta^{\mu \nu} \frac{\left(\partial \nu_{0}\right) \cdot(x-Y(\tau))}{U \cdot(x-Y(\tau))}\right)_{\tau=\tau_{0}} .
\end{aligned}
$$

The retarded solution for $\bar{h}_{(0)}^{\mu \nu}$ is

$$
\bar{h}_{(0)}^{\mu \nu}=-\frac{\kappa^{2} M}{2 \pi}\left[\frac{U^{\mu}(\tau) U^{\nu}(\tau)}{U \cdot(x-Y(\tau))}\right]_{\tau=\tau_{0}} .
$$

Within our approximations, the only surviving terms in the equation for $\bar{h}_{(1)}^{\mu \nu}$ are the velocity fields of the scalar $\nu$. As in the case of the gauge field, the fluctuations $\bar{h}_{(1)}^{\mu \nu}$ are expected to contribute to the observed radiation field.

To confirm this expectation consider the gauge condition $\partial_{\mu} \bar{h}^{\mu \nu}=0$. For $\bar{h}_{(0)}^{\mu \nu}$ we have

$$
\partial_{\mu} \bar{h}_{(0)}^{\mu \nu}=-\frac{\kappa^{2} M}{2 \pi}\left[\frac{\dot{U}^{\nu}}{U \cdot(x-Y(\tau))}\right]_{\tau=\tau_{0}} .
$$


This has the correct $1 / R$ fall off behaviour for a first derivative of the graviton field to yield a non-vanishing energy flux at infinity. Using the equations of motion for the branes (2.29) and (2.31), we find that the gauge condition is satisfied if

$$
\partial_{\mu} \bar{h}_{(1)}^{\mu \nu}=-\frac{\kappa^{2} M}{2 \pi} A p\left[\frac{\partial^{\nu} \nu_{0}(Y(\tau))}{U \cdot(x-Y(\tau))}\right]_{\tau=\tau_{0}} .
$$

A hint for the above reasoning comes from consideration of the graviton equations of motion

$$
\partial^{2} \bar{h}^{\mu \nu}=-2 \kappa^{2}\left(T_{\text {source }}^{\mu \nu}+T_{\nu}^{\mu \nu}\right) .
$$

The conservation equation $\partial_{\mu} T^{\mu \nu}=0$ (which implies that $\partial^{2} \partial_{\mu} \bar{h}^{\mu \nu}=0$ ) is a necessary condition for $\partial_{\mu} \bar{h}^{\mu \nu}=0$ and, after some algebra, gives the brane equations of motion.

To summarise: we found the solution for $\bar{h}_{(0)}^{\mu \nu}$ and the differential equations obeyed by $\bar{h}_{(1)}^{\mu \nu}$, which can be integrated (at least numerically). Both terms are expected to contribute to the observed radiation. To obtain the total radiated power and other quantities of interest, the solution for the metric fluctuations can be substituted in the formula for the stress-energy tensor of the gravitational field around flat space, which is well-known in general relativity (see for example [32], chapter 7 for a discussion).

\section{Discussion}

In this paper, we have considered accelerated wrapped fundamental strings and $\mathrm{D} p$ branes in the linear approximation to the classical limit of superstring theory. The results we obtained are thus valid provided the string coupling $g_{s}$ is small and the magnitude of the acceleration $a \ll 1 / \sqrt{\alpha^{\prime}}$. We showed that such objects generically produce scalar, gauge field (the appropriate NS-NS or R-R field with respect to which the brane is charged) and gravitational radiation. The field responsible for the acceleration was taken to be an external scalar incident on the branes. The space-time in which the branes propagate was compactified to four dimensions on $T^{p} \times T^{(6-p)}$. We neglected both the Kałuża-Klein modes of a string or brane source around $T^{(6-p)}$ and the internal excitations of a $p$-brane wrapped around $T^{p}$. It would be interesting to see if the inclusion of these modes leads to distinctive features of the emitted radiation. Alternatively, one can compactify on $T^{p}$ and obtain results similar to the four dimensional ones considered here for wrapped $p$-branes in $D=(6-p)$ space-time dimensions.

In the case of the bulk scalars, we were able to solve the linearised field equations and obtained a Larmor-type formula for the total radiated power. The angular 
distribution of the emitted radiation was studied. Finally, the cross section for scattering of the incident scalar wave was calculated; this gives an estimate for the classical radii of the branes. In the case of the gauge and gravitational fields, we were not able to solve the linearised field equations in closed form because of the coupling of the accelerating scalar field to the various fields produced by the moving source. However, we obtained differential equations which can be integrated; from the solutions to these equations, one can in principle derive the total power radiated in the form of gauge field and gravitational waves and also the cross sections for scattering of the incident external perturbation.

Our results may be viewed as a generalization to string theory and $p$-branes (including D-branes) of previous work by cosmologists on radiation from accelerated cosmic strings (for a review and references, see [33]). The approach consists in solving the field equations of the Maxwell-Einstein-Higgs system, or a suitable supergravity generalization thereof, coupled to accelerated localised sources in the weak field approximation. The linearization of the field equations serves two purposes. First, it simplifies the otherwise formidable task of solving the system of coupled partial differential equations for the scalar, gauge field, gravitational and other fields in the problem. It also allows one to avoid discussing thorny issues which make their appearance beyond the linear approximation: for example, the full Einstein equations (2.25) in the presence of a sharply localised source involve products of $\delta$-functions which must be dealt with either using the theory of distributions or by attributing a small thickness to the source and taking an appropriate limit. The weak-field approximation is certainly valid for sources whose fields fall off sufficiently fast in the far away region, where the radiation is observed. The thin-source approximation is valid in the long wave-length limit of string theory, but will break down at high energies: there the dynamics is governed by the light open strings, which determine the effective thickness of the source.

Our approach has also neglected a number of dynamical effects which it would be interesting to include in the analysis. For example, we have not discussed the backreaction of the emitted radiation on the source: as energy and momentum are carried away by the emitted radiation, the solution describing the source and its energymomentum tensor will be modified. In addition, the emitted momentum will make the source recoil and accelerate. These effects should be of import in understanding the dynamics and fate of strings and branes accelerated in a curved background, like that of a non-extremal black hole. In the classical limit we have considered here, such effects could be studied by viewing the actions (2.12) or (2.15) for the brane sources as probe actions [16], substituting the pull-backs of the corresponding 
space-time metrics and solving the corresponding field equations in the weak-field approximation (which will be valid as long as the probe and the 'heavy' object responsible for the acceleration are well separated).

It would also be very interesting to understand accelerated branes and radiation effects from branes in the context of the AdS/CFT correspondence [34, 35, 36]. In [37, the interactions of probe D3 branes in flat and in $A d S_{5} \times S_{5}$ backgrounds were considered. In the latter case, the effects of exchange of bulk supergravity modes carrying momentum in the brane directions were related to certain terms involving derivatives of the field strength in the action of the dual gauge theory. It is plausible that such terms, along with other higher loop terms in the gauge theory, correspond to accelerated probes in the curved background. Although the acceleration terms in the gauge theory discussed in [37] correspond in the bulk to NS-NS and R-R 2-form exchange, there should be additional terms corresponding to exchange of scalar and gravitational fields. It will be interesting to see whether the emission of radiation in the bulk can be computed in the gauge theory from these terms.

Another challenge to which our work points is of course to reproduce and obtain quantum corrections to results such as (3.17) and (3.18) on the radiation emitted by accelerated strings and $p$-branes directly from the underlying string theory. With current methods, this appears to be a rather daunting task: even for open F-strings ending on the D-branes (let alone NS fivebranes), the boundary conditions corresponding to an accelerated brane break the world-sheet conformal invariance. Clearly, some understanding of accelerated open string dynamics at the quantum level is required.

\section{Acknowledgements}

We would like to thank Costas Bachas, Michael Green, Michael Gutperle, Daniel Holz, Hermann Nicolai, Lori Paniak and Malcolm Perry for helpful comments. The work of MSC was supported by FCT (Portugal) under the PRAXIS XXI programme and by NSF grant PHY-9802484.

\section{References}

[1] C. Callan, J. Harvey and A. Strominger, Supersymmetric String Solitons, hepth/9112030 and references therein. 
[2] P. K. Townsend, The Eleven-Dimensional Supermembrane Revisited, Phys. Lett. B350 (1995) 184, hep-th/9501068.

[3] C. M. Hull, Gravitational Duality, Branes, and Charges, Nucl. Phys. B509 (1998) 216, hep-th/9705162.

[4] J. Polchinski, Dirichlet Branes and Ramond-Ramond Charges, Phys. Rev. Lett. 75 (1995) 184, hep-th/9510017.

[5] J. Polchinski, String Theory, Cambridge University Press, Cambridge (1998), chapter 13.

[6] A. Sen, Non-BPS States and Branes in String Theory, hep-th/9904207 and references therein.

[7] R. Dijkgraaf, E. Verlinde and H. Verlinde, BPS Spectrum of the Five-Brane and Black Hole Entropy, Nucl. Phys. B486 (1997) 77, hep-th/9603126; BPS Quantization of the Five-Brane, Nucl. Phys. B486 (1997) 89, hep-th/9604055; Notes on Matrix and Micro Strings, Nucl. Phys. Proc. Suppl. 62 (1998), hepth/9709107.

[8] I. R. Klebanov and L. Thorlacius, The Size of D-Branes, Phys. Lett. B371 (1996) 51, hep-th/9510200.

[9] J. L. F. Barbón, D-Brane Form Factors at High Energy, Phy. Lett. B382 (1996) 60, hep-th/9601098.

[10] S. S. Gubser, A. Hashimoto, I. R. Klebanov and J. M. Maldacena, Gravitational Lensing by p-Branes, Nucl. Phys. B472 (1996) 231, hep-th/9601057.

[11] M. R. Garousi and R. C. Myers, Superstring Scattering from D-Branes, Nucl. Phys. B475 (1996) 193, hep-th/9603194.

[12] A. Hashimoto and I. R. Klebanov, Decay of Excited D-Branes, Phys. Lett. B381 (1996) 437, hep-th/9604065.

[13] C. Bachas, D-Brane Dynamics, Phys. Lett. B374 (1996) 37, hep-th/9511043.

[14] S. H. Shenker, Another Length Scale in String Theory?, hep-th/9509132.

[15] M. R. Douglas, D. Kabat, P. Pouliot and S. H. Shenker, D-Branes and Short Distances in String Theory, Nucl. Phys. B485 (1997) 85, hep-th/9608024. 
[16] M. R. Douglas, J. Polchinski and A. Strominger, Probing Five-Dimensional Black Holes with D-Branes, JHEP 12 (1997) 3, hep-th/9703031.

[17] V. Periwal, O. Tafjord, D-Brane Recoil, Phys. Rev. D54 (1996) 3690, hepth/9603156.

[18] W. Fischler, S. Paban, M. Rozali, Collective Coordinates for D-Branes, Phys. Lett. B381 (1996) 62, hep-th/9604014.

[19] V. Periwal, R. von Unge, Accelerating D-Branes, Phys. Lett. B430 (1998) 71, hep-th/9801121.

[20] V. P. Frolov and N. Sánchez, Instability of Accelerated Strings and the Problem of Limiting Acceleration, Nucl. Phys. B439 (1991) 815.

[21] E. Witten, Bound States of Strings and p-Branes, Nucl. Phys. B460 (1996) 335, [hep-th/9510135.

[22] J. H. Schwarz, An SL(2,Z) Multiplet of Type II Superstrings, Phys. Lett. B360 (1995) 13, hep-th/9508143; Erratum: ibid, B364 (1995) 252.

[23] J. Maharana and J. H. Schwarz, Noncompact Symmetries in String Theory, Nucl. Phys. B390 (1993) 3, hep-th/9207016.

[24] A. Dabholkar, G. W. Gibbons, J. A. Harvey and F. Ruiz-Ruiz, String Solitons, Nucl. Phys. B340 (1990) 33.

[25] G. Horowitz and A. Strominger, Black Strings and p-Branes, Nucl. Phys. B360 (1991) 197.

[26] M. R. Douglas, Branes within Branes, hep-th/9512077.

[27] M. B. Green, C. M. Hull and P. K. Townsend, D-Brane Wess-Zumino Actions, T-Duality and the Cosmological Constant, Phys. Lett. B382 (1996) 65, hepth/9604119.

[28] M. B. Green, J. A. Harvey and G. Moore, I-Brane Inflow and Anomalous Couplings on D-Branes, Class. Quant. Grav. 14, 47 (1997), hep-th/9605033.

[29] C. P. Bachas, P. Bain and M. B. Green, Curvature Terms in D-Brane Actions and their M-theory Origin, JHEP 05 (1999) 011, hep-th/9903210.

[30] J. D. Jackson, Classical Electrodynamics, Second Edition, John Wiley \& Sons, New York (1975). 
[31] L. D. Landau and E. M. Lifshitz, The Classical Theory of Fields, Third English Edition, Pergamon, Oxford and Addison-Wesley, Reading, Mass. (1971).

[32] S. Weinberg, Gravitation and Cosmology: Principles and Applications of the General Theory of Relativity, John Wiley \& Sons, New York (1972).

[33] A. Vilenkin and E. Shellard, Cosmic Strings and Other Topological Defects, Cambridge University Press, Cambrige (1994), chapters 7 and 8.

[34] J. Maldacena, The Large N Limit of Superconformal Field Theories and Supergravity, Adv. Theor. Math. Phys. 2 (1998) 231, hep-th/9711200.

[35] S. S. Gubser, I. R. Klebanov and A. M. Polyakov, Gauge Theory Correlators from Noncritical String Theory, Phys. Lett. B428 (1998) 105, hep-th/9802109.

[36] E. Witten, Anti-De Sitter Space and Holography, Adv. Theor. Math. Phys. 2 (1998) 253, hep-th/9802150.

[37] S. R. Das, Brane Waves, Yang-Mills Theories and Causality, JHEP 02, 012 (1999), hep-th/9901004; Holograms of Branes in the Bulk and Acceleration Terms in SYM Effective Actions, JHEP 9906 (1999) 029, hep-th/9905037. 